\title{
Acúmulo de macronutrientes na soja influenciado pelo cultivo prévio do capim-marandu, correção e compactação do solo ${ }^{1}$
}

\author{
Macronutrient accumulation in the soybean influenced by prior cultivation of \\ Marandu grass and soil remediation and compaction
}

\author{
Marcos André Silva Souza ${ }^{2}$, Valdemar Faquin ${ }^{3}$, Douglas Ramos Guelfi³*, Geraldo César de Oliveira ${ }^{3}$ e Carla \\ Elisa Alves Bastos 4
}

\begin{abstract}
RESUMO - O presente trabalho foi desenvolvido com o objetivo de avaliar o efeito do cultivo prévio do capim-Marandu (Brachiaria brizantha cv. Marandu), da aplicação de corretivos e da compactação do solo no acúmulo de macronutrientes pela soja cultivada em sucessão. O delineamento experimental foi inteiramente casualizado em esquema fatorial 4 x 6 x 2, com três repetições. Os fatores de estudo foram quatro densidades do solo: 1,0;1,20;1,40 e 1,60 Mg m³; seis tratamentos de correção: 1) controle, sem correção; 2) calcário; 3) silicato de cálcio; 4) gesso; 5) calcário + gesso; 6) silicato de cálcio + gesso; além de dois sistemas de cultivo: com e sem cultivo prévio do capim-Marandu. As unidades experimentais foram compostas por vasos de tubos de PVC de $20 \mathrm{~cm}$ de diâmetro, compostos por dois anéis: o anel inferior, de $40 \mathrm{~cm}$ de altura, recebeu o solo sob condições naturais e densidade de $1,0 \mathrm{Mg} \mathrm{m}^{-3}$; o anel superior, com $20 \mathrm{~cm}$ de altura representando $6,28 \mathrm{dm}^{3}$, recebeu os tratamentos de densidades, correção e gesso como descrito adiante. Em cada um foram conduzidas três plantas de soja (cv. Conquista) até o final do ciclo, quando o acúmulo de macronutrientes pela cultura foi avaliado. Os resultados mostraram que o cultivo prévio do capim-Marandu e o uso de corretivos amenizaram os efeitos negativos da compactação do solo sobre a nutrição da soja. A utilização de corretivos do solo contribuiu para o aumento no acúmulo de macronutrientes na parte aérea da soja, porém o incremento na compactação diminuiu o acúmulo de $\mathrm{N}, \mathrm{P}, \mathrm{K}, \mathrm{Ca}, \mathrm{Mg}$ e $\mathrm{S}$. A compactação do solo persistiu parcialmente mediante o cultivo prévio com o capim marandu.
\end{abstract}

Palavras-chave: Calcário. Densidade do solo. Gesso agrícola. Silicato de cálcio. Sistema de cultivo.

\begin{abstract}
The present work was developed with the objective of evaluating the effect of the prior cultivation of marandu grass (Brachiaria brizantha $\mathrm{cv}$. Marandu), conditioning applications and soil compaction on macronutrient accumulation in soybeans grown under successive cultivation. The experimental design was completely randomized in a 4 × 6 × 2 factorial layout with three replications. The factors under study were four soil densities: 1.0, 1.20,1.40 and 1.60 $\mathrm{Mg} \mathrm{m}^{-3}$; six conditioning treatments: 1) a control with no conditioning, 2) limestone; 3) calcium silicate, 4) gypsum, 5) limestone and gypsum, 6) calcium silicate and gypsum; besides two cultivation systems: with and without the prior cultivation of marandu grass. The experimental units were made from PVC tubes, $20 \mathrm{~cm}$ in diameter, composed of two rings: the lower ring, $40 \mathrm{~cm}$ in height, received soil under natural conditions and at a density of $1.0 \mathrm{Mg} \mathrm{m}$ 3; the upper ring, $20 \mathrm{~cm}$ in height, representing $6.28 \mathrm{dm}^{3}$, received the density, conditioning and gypsum treatments as described below. In each unit, three soybean plants (cv. Conquest) were grown until the end of their cycle, when the accumulation of macronutrients in the crop was evaluated. The results showed that prior cultivation of marandu grass and the use of conditioners mitigated the negative effects of soil compaction on soybean nutrition. The use of soil conditioners contributed to an increase in the accumulation of nutrients in the soybean shoot, but the increase in compaction reduced the accumulation of $\mathrm{N}, \mathrm{P}, \mathrm{K}, \mathrm{Ca}, \mathrm{Mg}$ and $\mathrm{S}$. Soil compaction persisted in part through the prior cultivation of Marandu grass.
\end{abstract}

Key words: Calcium silicate. Cropping systems. Lime. Phosphogypsum. Soil bulk density.

\footnotetext{
*Autor para correspondência

${ }^{1}$ Recebido para publicação em 01/03/2011; aprovado em 06/03/2012

Parte da Tese de Doutorado do primeiro autor apresentada ao Programa de Pós-graduação em Ciência do Solo da Universidade Federal de Lavras; Pesquisa realizada com recursos financeiros da FAPEMIG e do CNPq

${ }^{2}$ Universidade de Rio Verde/FESURV, Fazenda Fontes do Saber, Rio Verde-GO, Brasil, 75.901-970, marcosandre@ fesurv.br

${ }^{3}$ Universidade Federal de Lavras/UFLA, Lavras-MG, Brasil, 37.200-000, douglasguelfi@dcs.ufla.br, vafaquin@dcs.ufla.br, geraldooliveira@dcs.ufla.br

${ }^{4}$ Universidade de São Paulo/USP/Esalq, Piracicaba-SP, Brasil, 13.418-900, carlinhabastos@ hotmail.com
} 


\section{INTRODUÇÃO}

$\mathrm{Na}$ agricultura moderna o uso frequente de máquinas e implementos agrícolas pesados, com o tempo, inevitavelmente resulta na compactação do solo. Esta compactação promove alterações nos atributos físicos resultando em aumento da densidade, resistência à penetração de raízes e diminuição da infiltração de água (ALAKUKKU; WEISSKKOPF; CHAMEN, 2003), comprometendo assim, o crescimento e a nutrição de plantas. Portanto, é muito importante o estudo de práticas e alternativas que aliviem ou minimizem os efeitos da compactação.

Segundo Silva e Cabeda (2006) os diferentes sistemas de manejo do solo levam a diversos resultados quanto à compactação. Seus efeitos sobre os atributos físicos podem ser bastante diferenciados (AZEVEDO et al., 2007; KLEIN; LIBARDO, 2002; SOUZA; ALVES, 2003) isto porque dependem do tipo de preparo realizado, da intensidade de movimentação, trânsito de máquinas e do manejo dos resíduos vegetais (MATIAS, 2009).

Nesse contexto, destaque é dado aos sistemas de produção como o plantio direto e a integração lavoura-pecuária. O uso de rotação e sucessão de culturas promove melhor aproveitamento dos insumos tornando esses sistemas mais eficientes (SIQUEIRA JÚNIOR, 2005), além de aumentar a fertilidade do solo, ciclagem de nutrientes e recuperação da estrutura do solo (GARCIA et al., 2004).

Além do sistema de manejo, a adição de corretivos pode promover alterações benéficas aos atributos físicos (CORRÊA et al., 2009) e químicos do solo (ZAMBRANO et al., 2003) como aumento da disponibilidade de $\mathrm{P}$ e redução da toxidez por $\mathrm{Al}$ e Mn (BULL; CRUSCIOL, 2004; CAIRES et al., 2008; CARVALHO-PUPPATTO) em solos onde é necessária a correção da acidez. As alterações nos atributos físicos do solo ocorrem devido ao fato de os corretivos possuírem $\mathrm{Ca}$ e $\mathrm{Mg}$ em sua composição e estes elementos atuam como ligantes entre as partículas da fase sólida do solo, permitindo maior agregação (OLIVEIRA, 2008). Além dos efeitos diretos, a utilização de corretivos também tem efeitos indiretos por aumentar a produção de fitomassa das culturas, aumentar o teor de matéria orgânica do solo e por favorecera atividade microbiana, fatores que contribuem para o aumento da agregação do solo (CASTRO FILHO; MUZILLI; PODANOSCHI, 1998; SANTOS; CAMARGO, 1999).

Atualmente a correção do solo é realizada com uso de calcário seja ele dolomítico, calcítico ou magnesiano. Recentemente os silicatos de cálcio e magnésio são utilizados como corretivos de solo aumentando a produtividade das culturas, em especial as acumuladoras de silício como o arroz (PEREIRA, 2004). Embora não seja um corretivo de solo, o emprego do gesso agrícola melhora o ambiente radicular em profundidade, diminuindo a toxidez por alumínio e fornecendo cálcio e enxofre ao sistema radicular, potencializando maior absorção de água e nutrientes e refletindo em maior crescimento e produção das culturas.

Dessa forma, o objetivo desse trabalho foi avaliar o efeito do cultivo prévio do capim-Marandu (Brachiaria brizantha cv. Marandu) e do uso de corretivos do solo sobre os efeitos da compactação no acúmulo de macronutrientes pela soja cultivada em sucessão.

\section{MATERIAL E MÉTODOS}

O experimento foi conduzido em casa de vegetação do Departamento de Ciência do Solo (DCS) da Universidade Federal de Lavras (UFLA), utilizando amostras do horizonte B de um Latossolo Vermelho Distrófico textura argilosa, coletado no campus da UFLA, Lavras - MG, sob vegetação natural. Após secas ao ar e peneiradas em malha de dois milímetros, subamostras foram tomadas para caracterizações físicas e químicas (EMPRESA BRASILEIRA DE PESQUISA AGROPECUÁRIA, 1999): $\mathrm{pH}\left(\mathrm{H}_{2} \mathrm{O}\right)=4,4 ; \mathrm{M} . \mathrm{O}=0,3 \mathrm{~g} \mathrm{~kg}^{-1} ; \mathrm{K}^{+}=19 \mathrm{mg} \mathrm{dm}^{-3}$; $\mathrm{S}=10 \mathrm{mg} \mathrm{dm}^{-3} ; \mathrm{P}($ Mehlich 1$)=0,7 \mathrm{mg} \mathrm{dm}^{-3} ; \mathrm{Ca}^{2+}=$ $0,1 \mathrm{cmol}_{\mathrm{c}} \mathrm{dm}^{-3} ; \mathrm{Mg}^{2+}=0,1 \mathrm{cmol}_{\mathrm{c}} \mathrm{dm}^{-3} ; \mathrm{Al}^{3+}=0,8 \mathrm{cmol}_{\mathrm{c}}$ $\mathrm{dm}^{-3} ; \mathrm{H}+\mathrm{Al}=7,0 \mathrm{cmol}_{\mathrm{c}} \mathrm{dm}^{-3} ; \mathrm{SB}=0,3 \mathrm{cmol}_{\mathrm{c}} \mathrm{dm}^{-3} ; \mathrm{t}=$ $1,1 \mathrm{cmol}_{\mathrm{c}} \mathrm{dm}^{-3} ; \mathrm{T}=7,3 \mathrm{cmolc} \mathrm{dm}^{-3} ; \mathrm{m}=76 \% ; \mathrm{Fe}^{2+}=35$ $\mathrm{mg} \mathrm{dm}{ }^{-3} ; \mathrm{Zn}^{2+}=1,0 \mathrm{mg} \mathrm{dm}^{-3} ; \mathrm{Cu}^{2+}=1,8 \mathrm{mg} \mathrm{dm}^{-3} ; \mathrm{Mn}^{2+}$ $=10,2 \mathrm{mg} \mathrm{dm}^{-3} ;$ Areia $=210 \mathrm{~g} \mathrm{~kg}^{-1} ;$ Silte $=90 \mathrm{~g} \mathrm{~kg}^{-1}$; Argila $=700 \mathrm{~g} \mathrm{~kg}^{-1} ; \mathrm{SiO}_{2}=129,8 \mathrm{~g} \mathrm{~kg}^{-1} ; \mathrm{Al}_{2} \mathrm{O}_{3}=319,1$ $\mathrm{g} \mathrm{kg}^{-1} ; \mathrm{Fe}_{2} \mathrm{O}_{3}=171,8 \mathrm{~g} \mathrm{~kg}^{-1} ; \mathrm{TiO}_{2}=22,0 \mathrm{~g} \mathrm{~kg}^{-1} ; \mathrm{Ki}=$ 0,$41 ; \mathrm{Kr}=0,26$.

O cultivo prévio do capim-Marandu foi conduzido em vasos (colunas) de PVC de $20 \mathrm{~cm} \mathrm{de}$ diâmetro, compostos por dois anéis: o anel inferior, de $40 \mathrm{~cm}$ de altura, recebeu o solo sob condições naturais e densidade de $1,0 \mathrm{Mg} \mathrm{m}^{-3}$; o anel superior, com $20 \mathrm{~cm}$ de altura, recebeu os tratamentos de densidades e de correção do solo como descrito adiante. Os tratamentos de correção e compactação foram aplicados em seis repetições, das quais, três foram cultivadas com o capim-Marandu e três permaneceram em pousio, com umidade na capacidade de campo, visando estabelecer os sistemas de cultivo do presente trabalho com soja. 
Os tratamentos de densidades do solo foram: 1,$0 ; 1,20 ; 1,40$ e $1,60 \mathrm{Mg} \mathrm{m}^{-3}$ e os tratamentos relativos à correção do solo foram: 1) controle (sem correção); 2) calcário; 3) silicato de cálcio; 4) gesso; 5) calcário + gesso e 6) silicato + gesso.

O calcário utilizado foi formado pela mistura de carbonato de cálcio e carbonato de magnésio p.a, na equivalência de Ca:Mg de 4:1. A dose de calcário aplicada foi estimada pelo método de neutralização de $\mathrm{Al}^{+3}$ e da elevação dos teores de $\mathrm{Ca}^{+2}$ e $\mathrm{Mg}^{+2}$ e a dose de gesso $\left(\mathrm{CaSO}_{4} 2 \mathrm{H}_{2} \mathrm{O}\right.$ p.a.) foi estimada com base na textura do solo, de acordo com Ribeiro, Guimarães e Alvarez (1999). A dose de silicato de cálcio p.a. foi determinada considerando a equivalência do óxido de cálcio do silicato e do calcário.

Os tratamentos envolvendo o calcário e o silicato foram misturados ao volume de solo do anel superior para os respectivos vasos e incubados por 45 dias antes da aplicação dos tratamentos de compactação. O tratamento envolvendo apenas o gesso foi aplicado na superfície dos vasos após a compactação do solo e ficaram 30 dias em repouso com umidade na capacidade de campo.

Antes da aplicação dos tratamentos de compactação, macro e micronutrientes foram misturados nas amostras do anel superior de todos os tratamentos de acordo com MALAVOLTA (1980), com o uso de fontes p.a. Para a aplicação dos tratamentos de compactação, a umidade do solo foi corrigida para $15 \%$. Na compactação, foi utilizada uma prensa hidráulica e para evitar deformações ou rompimento do tubo do vaso de PVC pela compressão do solo, o anel de PVC foi acomodado dentro de outro anel metálico (chapa de aço 3/8”) de iguais dimensões.

Das seis repetições de cada tratamento, em três foram cultivadas cinco plantas de capim-Marandu (Brachiaria brizantha cv. Marandu), que sofreram três cortes a cinco centímetros da superfície do solo a intervalos de 30 dias. Antes do semeio da soja, após o último corte do capim-Marandu, o mesmo permaneceu em rebrota por 15 dias, quando se realizou a dessecação com glyfosato na dose de quatro litros por hectare. A massa seca produzida foi quantificada e picada em pedaços de três a cinco centímetros e depositada na superfície dos vasos correspondentes, na dose de 6,4 $\mathrm{t} \mathrm{ha}^{-1}\left(22,16 \mathrm{~g} \mathrm{vaso}^{-1}\right)$, próxima da usada por Corrêa, Mauad e Rosolem (2004) de 8,0 t ha-1. Esses vasos permaneceram em pousio por 60 dias com umidade na capacidade de campo e receberam, antes do plantio da soja, uma adubação básica de macro e micronutrientes na forma de solução nutritiva, com $50 \%$ da dose recomendada por Malavolta (1980), visando repor o extraído pela parte aérea colhida do capim-Marandu.

Em seguida, tanto nos vasos cultivados com capim-Marandu (sistema cultivado) quanto nos que permaneceram em pousio (sistema não cultivado), semearam-se cerca de 10 sementes de soja (Glycine $\operatorname{Max} \mathrm{L}, \mathrm{cv}$. Conquista) por vaso, desbastando-se para três plântulas 10 dias após a emergência. Irrigações diárias foram realizadas com água deionizada visando manter a umidade do solo próxima da capacidade de campo. Coberturas nitrogenadas e potássicas também foram realizadas durante o período de cultivo da soja, em ambos os sistemas, de acordo com Malavolta (1980).

No final do ciclo da cultura, em ambos os sistemas de cultivo, as plantas foram colhidas, sendo a parte aérea e grãos secos em estufa a $65-70{ }^{\circ} \mathrm{C}$ e tomados os pesos da massa seca. $\mathrm{O}$ material vegetal foi moído e analisado para os macronutrientes de acordo com a metodologia descrita por Malavolta (1997). Nesse trabalho, os dados de produção de massa seca, cujos resultados estão em Souza (2011), foram usados apenas para relacioná-los com a concentração dos macronutrientes e calcular seus acúmulos na parte aérea da soja.

Os dados foram submetidos à análise de variância e o desdobramento das interações significativas por meio de regressão para o fator densidade utilizando o software SISVAR 4.3 (FERREIRA, 2000).

\section{RESULTADOS E DISCUSSÃO}

Primeiramente é necessário enfatizar que devido ao pequeno crescimento e morte das plântulas, não foi possível no tratamento controle, sem cultivo, a obtenção dos dados das variáveis propostas no trabalho. Dessa forma, o tratamento controle, tanto com quanto sem o cultivo prévio do capim-marandu, não foi incluído na análise estatística.

Os sistemas de cultivo (com e sem cultivo prévio do capim-Marandu), as densidades do solo, os tratamentos de correção bem como a interação entre esses fatores influenciaram significativamente $(\mathrm{P}<0,05)$ o acúmulo de macronutrientes pela soja (Figura 1).

Na Figura 1, cujas equações de regressão são apresentadas na Tabela 1 , vê-se que o cultivo prévio do capim-Marandu, independente dos tratamentos de correção, promoveu aumentos significativos no acúmulo dos macronutrientes pela soja, à exceção dos acúmulos de Ca (Figura 1d) e de Mg (Figura 1e) nas 
Figura 1 - Acúmulo de nitrogênio (a), fósforo (b), potássio (c), cálcio (d), magnésio (e) e enxofre (f) na parte área da soja cultivada em vasos com e sem cultivo prévio do capim-marandu em função das densidades do solo
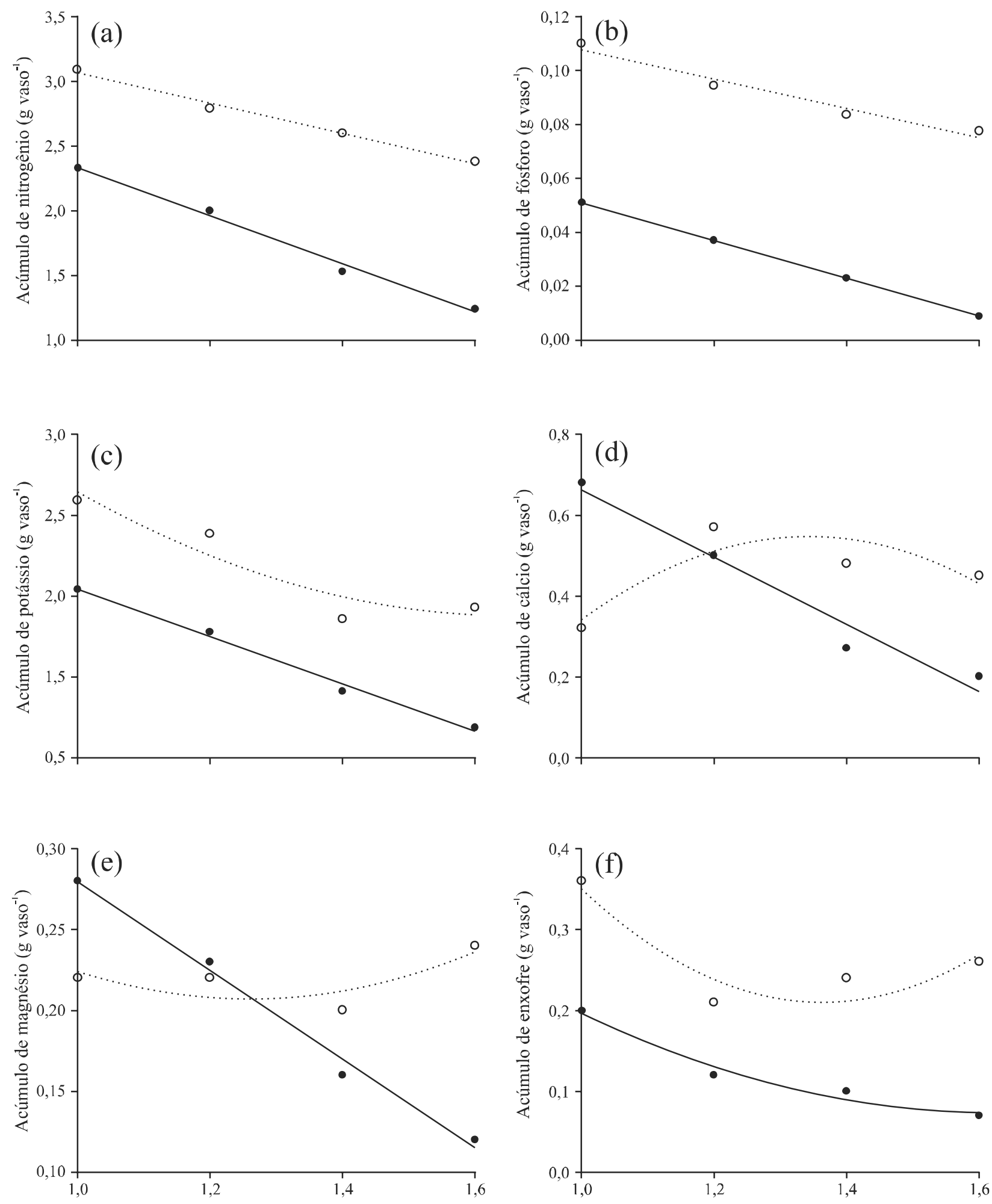

- Densidade do solo $\left(\mathrm{mg} \mathrm{m}^{-3}\right)$
Sem cultivo

Densidade do solo $\left(\mathrm{mg} \mathrm{m}^{-3}\right)$

- Com cultivo 
Tabela 1 - Equações de regressão para o acúmulo de nitrogênio, fósforo e potássio na parte aérea do capim-marandu e os valores estimados de cada variável para as densidades de 1,0 e $1,60 \mathrm{Mg} \mathrm{m}^{-3}$ e o percentual de redução da densidade 1,60 em relação $1,0 \mathrm{Mg} \mathrm{m}^{-3}$

\begin{tabular}{|c|c|c|c|c|c|}
\hline \multirow{2}{*}{ Sistemas } & \multirow{2}{*}{ Equação } & \multirow{2}{*}{$\mathrm{R}^{2}$} & \multicolumn{2}{|c|}{ Densidades $\left(\mathrm{Mg} \mathrm{m}^{-3}\right)$} & \multirow{2}{*}{$\frac{\text { Redução }}{(\%)}$} \\
\hline & & & 1,00 & 1,60 & \\
\hline \multicolumn{6}{|c|}{ Nitrogênio (g vaso-1) } \\
\hline Sem cultivo & $Y=4,223-1,16 x$ & $0,99 *$ & 3,1 & 2,3 & 26 \\
\hline Com cultivo & $Y=4,206-1,87 x$ & $0,99 *$ & 2,3 & 1,2 & 48 \\
\hline \multicolumn{6}{|c|}{ Fósforo (g vaso-1) } \\
\hline Sem cultivo & $Y=0,121-0,07 x$ & $0,98 *$ & 0,05 & 0,009 & 82 \\
\hline Com cultivo & $Y=0,1616-0,054$ & $0,96^{*}$ & 0,108 & 0,075 & 31 \\
\hline \multicolumn{6}{|c|}{ Potássio (g vaso ${ }^{-1}$ ) } \\
\hline Sem cultivo & $Y=3,638-1,83 x$ & $0,99 *$ & 1,8 & 0,7 & 61 \\
\hline Com cultivo & $Y=7,62-7,26 x+2,19 x^{2}$ & $0,89 *$ & 2,55 & 1,61 & 37 \\
\hline \multicolumn{6}{|c|}{ Cálcio (g vaso-1 $)$} \\
\hline Sem cultivo & $Y=1,498-0,83 x$ & $0,96 *$ & 0,66 & 0,16 & 76 \\
\hline Com cultivo & $Y=-2,61+4,7 x-1,75 x^{2}$ & $0,75^{*}$ & 0,34 & 0,43 & 26 \\
\hline \multicolumn{6}{|c|}{ Magnésio (g vaso-1) } \\
\hline Sem cultivo & $Y=0,555-0,27 x$ & $0,99 *$ & 0,16 & 0,12 & 25 \\
\hline Com cultivo & $Y=0,60-0,63 x+0,25 x^{2}$ & $0,60 *$ & 0,22 & 0,23 & 4 \\
\hline \multicolumn{6}{|c|}{ Enxofre $\left(\mathrm{g} \mathrm{vaso}^{-1}\right)$} \\
\hline Sem cultivo & $Y=0,389-0,20 x$ & $0,90 *$ & 0,18 & 0,06 & 67 \\
\hline Com cultivo & $Y=2,18-2,90 x+1,06 x^{2}$ & $0,85^{*}$ & 0,34 & 0,25 & 26 \\
\hline
\end{tabular}

*Significativo a $5 \%$ pelo teste $\mathrm{F}$

densidades 1,0 e 1,20 $\mathrm{Mg} \mathrm{m}^{-3}$, respectivamente. Em ambos os sistemas de cultivo, o aumento da densidade do solo promoveu uma diminuição significativa nos acúmulos de macronutrientes, à exceção para o do Ca e $\mathrm{Mg}$ no tratamento com cultivo prévio da gramínea.

De maneira geral, o acúmulo dos nutrientes nos vegetais é mais dependente da massa seca produzida do que de seus teores nos tecidos. Dessa maneira, o acúmulo dos macronutrientes (Figura 1) seguiu o mesmo comportamento do crescimento da soja em função dos sistemas de cultivos e das densidades do solo estudadas, cujos resultados estão em Souza (2011). Assim, os maiores acúmulos de macronutrientes foram observados na soja cultivada no solo com cultivo prévio do capim-Marandu e, em ambos os sistemas de cultivo, o aumento da densidade do solo promoveu reduções nos seus acúmulos, à exceção de $\mathrm{Ca}$ e $\mathrm{Mg}$.
Observa-se na Tabela 1, além das equações de regressão, os valores estimados das variáveis de acúmulo de macronutrientes para as densidades 1,0 e $1,60 \mathrm{Mg} \mathrm{m}^{-3}$ e o percentual de redução dessas variáveis na densidade $1,6 \mathrm{Mg} \mathrm{m}^{-3}$ em relação à $1,0 \mathrm{Mg} \mathrm{m}^{-3}$ para os sistemas sem e com cultivo prévio do capim-Marandu. Observase que a densidade de $1,6 \mathrm{Mg} \mathrm{m}^{-3}$ no solo sem cultivo foi altamente limitante à nutrição da soja, promovendo reduções no acúmulo de macronutrientes que variaram de $25 \%$ para o $\mathrm{Mg}$ e até $82 \%$ para o $\mathrm{P}$ no solo sem cultivo. Para o solo com cultivo, esses valores foram bem menores, variando de $4 \%$ no acúmulo de $\mathrm{Mg}$ até $27 \%$ no acúmulo de P. A alta impedância mecânica no solo compactado gera um ambiente desfavorável para o crescimento de plantas pela redução da aeração do solo e da disponibilidade de água e dos nutrientes (GOEDERT; SCHERMACK; FREITAS, 2002). 
Esses resultados mostram que o cultivo prévio do capim-Marandu foi uma prática bastante efetiva na amenização dos efeitos negativos da densidade sobre a nutrição da soja.

Analisando o efeito individual dos corretivos, observa-se que a diferença entre eles no acúmulo de N, Pe K (Figura 2) e de $\mathrm{Ca}, \mathrm{Mg}$ e S (Figura 3) foi pouco pronunciada no solo não cultivado previamente com o capim-Marandu e, em ambos os sistemas de cultivo, o acúmulo foi diminuído com a elevação dos valores das densidades do solo.

Os acúmulos de $\mathrm{N}$ e $\mathrm{K}$ (Figura 2) para ambos os sistemas de cultivo, seguiram basicamente a produção de massa seca pela soja (SOUZA, 2011). Para o Ca e o S (Figura 3), além da massa seca, os maiores acúmulos foram observados nos tratamentos nos quais estavam presentes no corretivo, principalmente calcário + gesso e silicato + gesso.

$\mathrm{O}$ acúmulo de $\mathrm{Mg}$ pela soja foi baixo com a aplicação do gesso em ambos os sistemas de cultivo (Figura $3 \mathrm{c}$, d). Isto pode ser atribuído ao fato do gesso ser somente fonte de cálcio e, possivelmente, devido a uma inibição competitiva do Ca do gesso com o $\mathrm{Mg}$ no processo de absorção (MALAVOLTA, 1980).

OpHéofator que mais influencia a disponibilidade e, em consequência, a absorção de nutrientes pelas plantas (SOUSA et al., 2007). Assim, no tratamento com gesso, o pH do solo permanecendo ácido, não favoreceu a disponibilidade e em consequência, o acúmulo dos macronutrientes, principalmente para o $\mathrm{P}$ nas menores densidades (Figura. $2 \mathrm{c}$, d). Em solos ácidos, ocorre menor disponibilidade de $\mathrm{P}$ pela sua precipitação com $\mathrm{Fe}$ e $\mathrm{Al}$, solúveis nessa condição de pH (SOUSA et al., 2007), reduzindo, assim, a sua absorção e acúmulo pela planta. Nas maiores densidades esse efeito não foi observado, devido à limitação física do solo ao crescimento das plantas, principalmente o radicular, não permitindo diferenças pronunciadas na produção de massa seca entre os corretivos utilizados (SOUZA, 2011).

No presente trabalho, embora de maneira pouco pronunciada, os corretivos com silício (silicato e silicato + gesso), promoveram maior acúmulo de $\mathrm{P}$, de forma mais acentuada no solo com cultivo (Figura 2 c, d). Pesquisas têm demonstrado que à semelhança do $\mathrm{P}$, o $\mathrm{Si}$ sofre a adsorção específica às superfícies dos óxidos de Fe e Al do solo (POZZA et al., 2007; SOUZA, 2011). Assim, o P e o Si competem entre si pelos mesmos sítios de adsorção, de maneira que o segundo pode deslocar o primeiro e vice-versa, da fase sólida para a líquida do solo (CARVALHO et al., 2000). De acordo com esses autores, o aumento do $\mathrm{pH}$ do solo pela aplicação do silicato, com a consequente insolubilização do $\mathrm{Fe}$ e $\mathrm{Al}$, reduz a precipitação do $\mathrm{P}$ com esses cátions, favorecendo a disponibilidade do $\mathrm{P}$ e a sua absorção pelas plantas.

As Tabelas 2 e 3 mostram as equações de regressão e, nas últimas três colunas, são apresentados os valores estimados dos acúmulos pela soja de $\mathrm{N}, \mathrm{Pe} \mathrm{K} \mathrm{e} \mathrm{Ca,} \mathrm{Mg} \mathrm{e} \mathrm{S,}$ respectivamente, para as densidades de 1,0 e $1,6 \mathrm{Mg} \mathrm{dm}^{-3} \mathrm{e}$ o percentual de redução da maior para a menor densidade, para todos os corretivos e sistemas de cultivo. Observase que, com exceção do Ca (Tabela 3 ) no sistema com cultivo, a densidade de $1,6 \mathrm{Mg} \mathrm{m}^{-3}$ reduziu sensivelmente o acúmulo dos nutrientes para todos os tratamentos de corretivos utilizados.

Chama à atenção o baixo acúmulo de $\mathrm{P}$ pela soja cultivada na densidade $1,6 \mathrm{Mg} \mathrm{m}^{-3}$ no solo sem cultivo (Tabela 2) e a grande redução em relação à densidade 1,0 $\mathrm{Mg} \mathrm{m}^{-3}$, com exceção do tratamento com gesso no qual a redução na produção ocasionada pela compactação, aliada à falta de correção da acidez, reduziu o acúmulo de P. Em solos compactados ocorre uma quebra de agregados e aumento na proporção de microporos e, assim, maior contato do fosfato com a superfície da fração sólida do solo, com o consequente aumento da fixação (NOVAIS; SMITH, 1999). Com isso, há menor disponibilidade, absorção e acúmulo de $\mathrm{P}$ pelas plantas. Além disso, o aumento da impedância mecânica reduz o crescimento radicular e a capacidade das raízes de absorver o nutriente, como verificado para milho (FOLONI; CALONEGO; LIMA, 2003; SHIERLAW; ALSTON, 1984).

Dessa forma, o menor volume de solo explorado pelas raízes e o movimento a curtas distâncias no solo, por difusão, fizeram que o $\mathrm{P}$ fosse o nutriente mais afetado pela compactação. Já no solo previamente cultivado pelo capim-marandu, as limitações físicas do solo ao crescimento da soja foram aliviadas e com isso, maior absorção de $\mathrm{P}$ e menor limitação da densidade $1,6 \mathrm{Mg} \mathrm{m}^{-3}$ no acúmulo do nutriente pelas plantas (Tabela 2).

SILVA e ROSOLEM (2001) verificaram reduções no acúmulo de potássio, cálcio e magnésio quando da elevação da densidade do solo de 1,12 para $1,36 \mathrm{Mg} \mathrm{m}^{-3}$ no cultivo da soja em sucessão a diferentes plantas de cobertura.

Em outro trabalho, CORRÊA et al. (2001) observaram reduções nas concentrações de N, P, K, $\mathrm{Mg}$ e $\mathrm{S}$ na parte aérea do cafeeiro com aumento da compactação do solo. Embora esses autores não tenham quantificado o acúmulo de nutrientes, o que evitaria o efeito diluição pelo crescimento vegetativo, os resultados encontrados vêm a corroborar os resultados obtidos nesse trabalho quanto aos efeitos negativo da compactação do solo na absorção de nutrientes. 
Figura 2 - Acúmulo de nitrogênio (a) e (b), fósforo (c) e (d) e potássio (e) e (f) na parte área da soja cultivada em vasos com e sem cultivo prévio do capim-marandu submetidos diferentes corretivos em função das densidades do solo
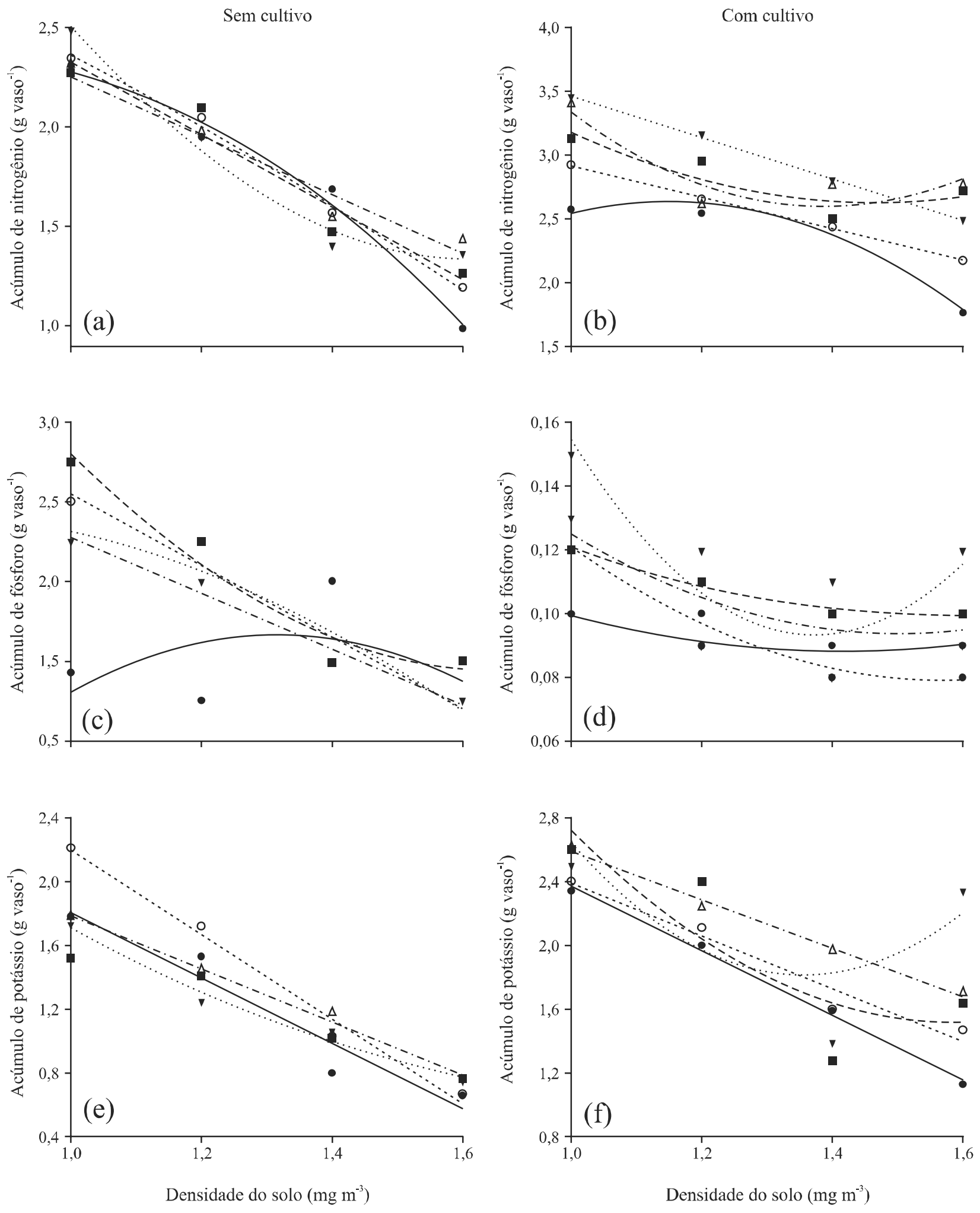

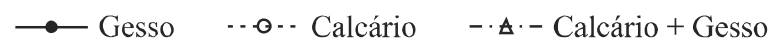


Figura 3 - Acúmulo de cálcio (a) e (b), magnésio (c) e (d) e enxofre (e) e (f) na parte área da soja cultivada em vasos com e sem cultivo prévio do capim-marandu submetidos diferentes corretivos em função das densidades do solo

Sem cultivo
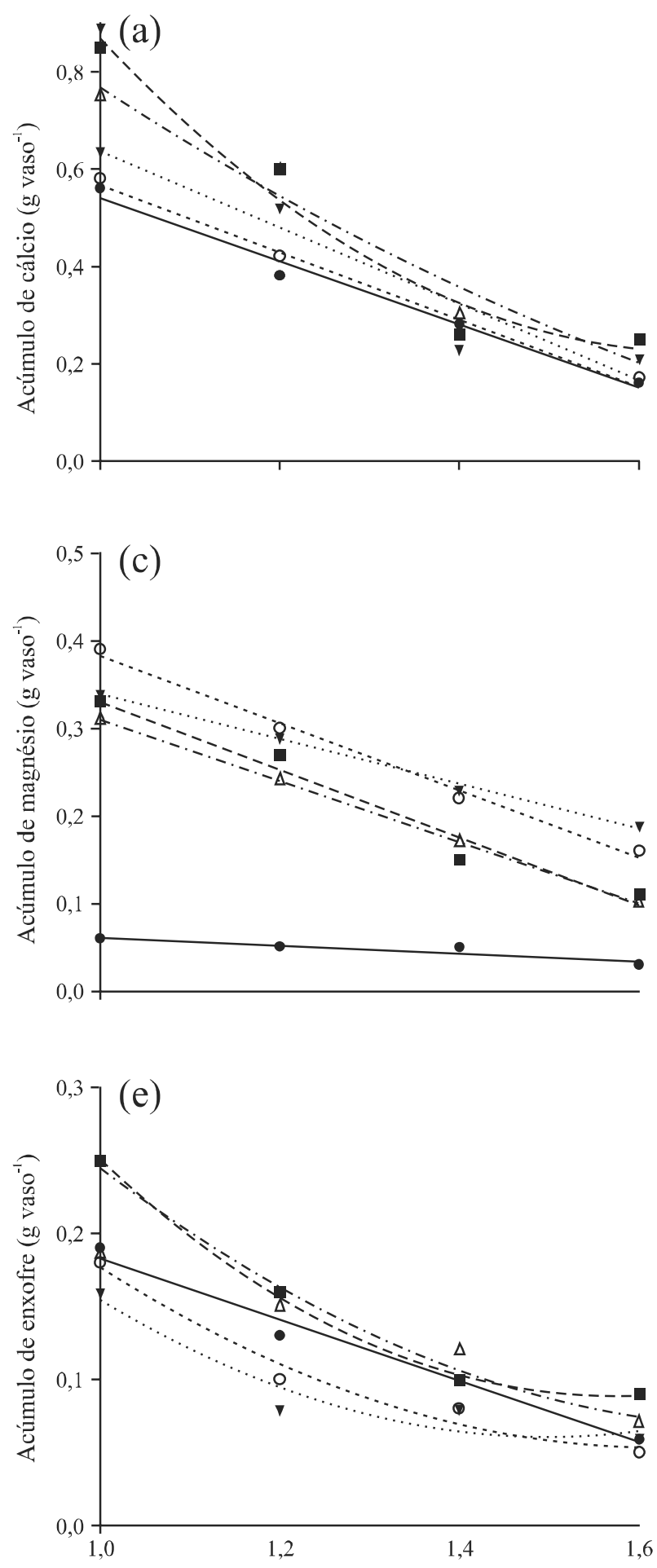

Densidade do solo $\left(\mathrm{mg} \mathrm{m}^{-3}\right)$

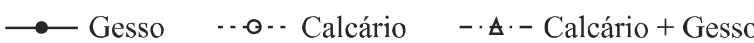
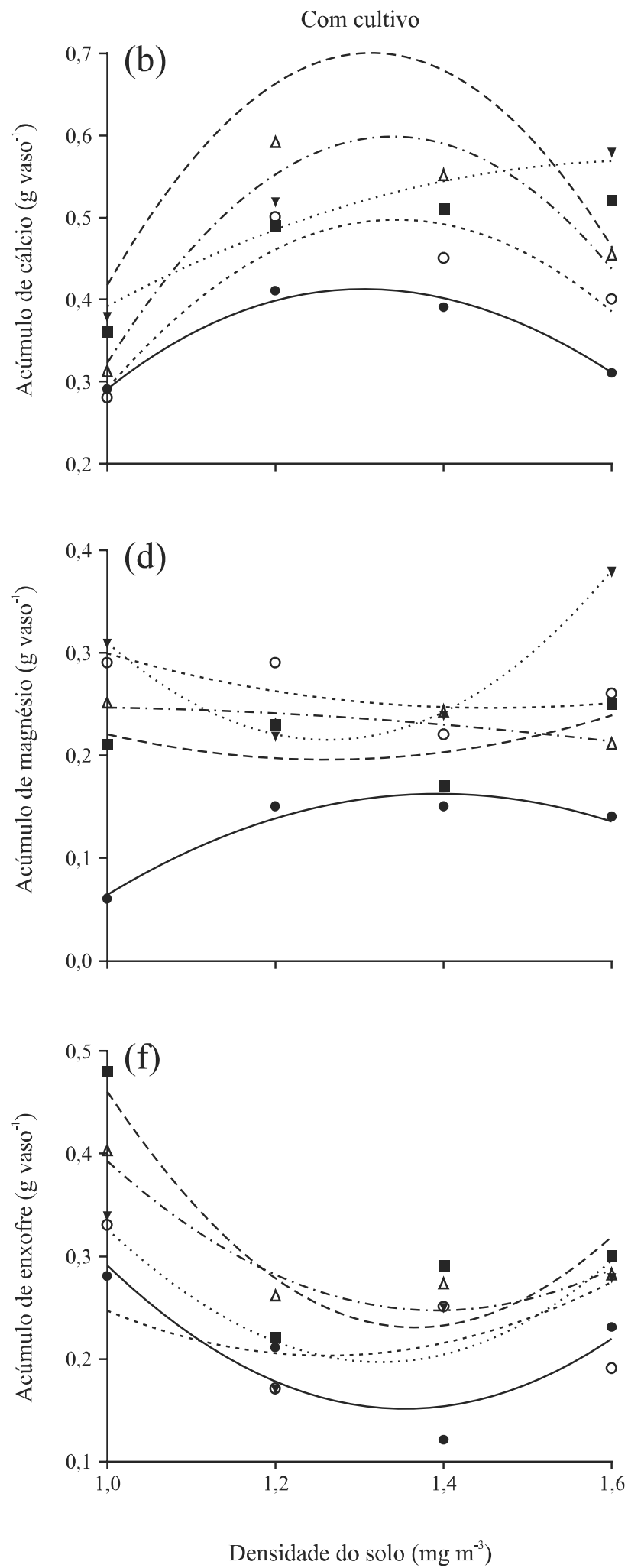

Silicato de Cálcio Silicato de Cálcio + Gesso 
Tabela 2 - Equações de regressão para o acúmulo de nitrogênio, fósforo e potássio na parte aérea da soja cultivada em vasos em função dos corretivos com e sem cultivo prévio do capim-marandu e os valores estimados de cada variável para as densidades de 1,0 e $1,60 \mathrm{Mg} \mathrm{m}^{-3}$ e o percentual de redução da densidade 1,6 em relação $1,0 \mathrm{Mg} \mathrm{m}^{-3}$

\begin{tabular}{|c|c|c|c|c|c|}
\hline \multirow{2}{*}{ Corretivos } & \multirow{2}{*}{ Equação } & \multirow{2}{*}{$\mathrm{R}^{2}$} & \multicolumn{2}{|c|}{ Densidades $\left(\mathrm{Mg} \mathrm{m}^{-3}\right)$} & \multirow{2}{*}{$\begin{array}{c}\text { Redução } \\
(\%)\end{array}$} \\
\hline & & & 1,00 & 1,60 & \\
\hline \multicolumn{6}{|c|}{ Acúmulo de nitrogênio $\left(\mathrm{g}\right.$ vaso $\left.^{-1}\right)$} \\
\hline \multicolumn{6}{|c|}{ Sem cultivo } \\
\hline Gesso & $Y=0,98^{*}+3,41 x-2,12 x^{2}$ & $0,98^{*}$ & 2,27 & 1,00 & 55 \\
\hline Calcário & $Y=3,62 *-0,83 x-0,44 x^{2}$ & $0,99 *$ & 2,35 & 1,17 & 50 \\
\hline Silicato & $Y=9,36^{*}-9,92 x+3,06 x^{2}$ & $0,98^{*}$ & 2,50 & 1,32 & 47 \\
\hline Calcário + gesso & $Y=5,56^{*}-4,39 x+1,13 x^{2}$ & $0,98^{*}$ & 2,30 & 1,43 & 37 \\
\hline Silicato + gesso & $Y=3,84 *-1,34 x-0,19 x^{2}$ & $0,95^{*}$ & 2,31 & 1,21 & 47 \\
\hline \multicolumn{6}{|c|}{ Com cultivo } \\
\hline Gesso & $Y=-2,90^{*}+9,63 x-4,19 x^{2}$ & $0,96^{*}$ & 2,54 & 1,78 & 29 \\
\hline Calcário & $Y=4,144 *-1,23 x$ & $0,99 *$ & 5,37 & 2,17 & 59 \\
\hline Silicato & $Y=4,87^{*}-1,30 x-0,13 x^{2}$ & $0,99 *$ & 3,44 & 2,46 & 28 \\
\hline Calcário + gesso & $Y=12,00^{*}-13,54 x+4,89 x^{2}$ & $0,84^{*}$ & 3,35 & 2,86 & 14 \\
\hline Silicato + gesso & $Y=8,01 *-7,34 x+2,50 x^{2}$ & $0,80^{*}$ & 3,17 & 2,67 & 15 \\
\hline \multicolumn{6}{|c|}{ Acúmulo de fósforo $\left(\mathrm{g}\right.$ vaso $\left.^{-1}\right)$} \\
\hline \multicolumn{6}{|c|}{ Sem cultivo } \\
\hline Gesso & $Y=-0,22+0,37 x-0,14 x^{2}$ & $0,22 *$ & 0,01 & 0,01 & 0 \\
\hline Calcário & $Y=0,15-0,09 x-4 e-16 x^{2}$ & $0,95^{*}$ & 0,06 & 0,003 & 95 \\
\hline Silicato & $Y=0,03+0,09 x-0,06 x^{2}$ & $0,90 *$ & 0,06 & 0,020 & 66 \\
\hline Calcário + gesso & $Y=0,12-0,07 x-2 E-16 x^{2}$ & $0,98 *$ & 0,05 & 0,008 & 84 \\
\hline Silicato + gesso & $Y=0,36-0,42 x+0,13 x^{2}$ & $0,96^{*}$ & 0,07 & 0,02 & 71 \\
\hline \multicolumn{6}{|c|}{ Com cultivo } \\
\hline Gesso & $Y=0,06 x 2-0,18 x+0,21$ & $0,93 *$ & 0,09 & 0,07 & 22 \\
\hline Calcário & $Y=0,13 \times 2-0,40 x+0,39$ & $0,98 *$ & 0,12 & 0,08 & 33 \\
\hline Silicato & $Y=0,44 x 2-1,20 x+0,92$ & $0,84^{*}$ & 0,16 & 0,13 & 18 \\
\hline Calcário + gesso & $Y=0,13 x 2-0,38 x+0,38$ & $0,55^{*}$ & 0,13 & 0,10 & 23 \\
\hline Silicato + gesso & $Y=0,06 x 2-0,20 x+0,26$ & $0,98^{*}$ & 0,12 & 0,09 & 25 \\
\hline \multicolumn{6}{|c|}{ Acúmulo de potássio $\left(\mathrm{g}\right.$ vaso $\left.^{-1}\right)$} \\
\hline \multicolumn{6}{|c|}{ Sem cultivo } \\
\hline Gesso & $Y=0,69 x 2-3,83 x+4,98$ & $0,94 *$ & 2,29 & 0,62 & 72 \\
\hline Calcário & $Y=0,82 x 2-4,77 x+6,20$ & $0,99 *$ & 2,25 & 0,67 & 70 \\
\hline Silicato & $Y=1,13 x 2-4,48 x+5,06$ & $0,98^{*}$ & 1,71 & 0,78 & 54 \\
\hline Calcário + gesso & $Y=-0,5 \times 2-0,36 x+2,63$ & $0,99 *$ & 1,77 & 0,77 & 56 \\
\hline Silicato + gesso & $Y=-0,89 x 2+0,95 x+1,46$ & $0,98^{*}$ & 1,52 & 0,70 & 53 \\
\hline \multicolumn{6}{|c|}{ Com cultivo } \\
\hline Gesso & $Y=-0,75 x 2-0,07 x+3,161$ & $0,99 *$ & 3,84 & 1,13 & 70 \\
\hline Calcário & $Y=x 2-4,25 x+5,68$ & $0,97 *$ & 2,43 & 1,44 & 40 \\
\hline Silicato & $Y=6,5 \times 2-17,64 x+13,78$ & $0,49 *$ & 2,64 & 2,20 & 16 \\
\hline Calcário + gesso & $Y=0,69 x 2-3,30 x+5,23$ & $0,99 *$ & 2,62 & 1,72 & 34 \\
\hline Silicato + gesso & $Y=3,5 \times 2-11,1 x+10,32$ & $0,75^{*}$ & 2,72 & 1,52 & 44 \\
\hline
\end{tabular}

*Significativo a $5 \%$ pelo teste $\mathrm{F}$ 
Tabela 3 - Equações de regressão para o acúmulo de cálcio, magnésio e enxofre na parte aérea da soja cultivada em vasos com e sem cultivo prévio do capim-marandu e os valores estimados de cada variável para as densidades de 1,0 e 1,60 $\mathrm{Mg} \mathrm{m}^{-3}$ e o percentual de redução da densidade 1,6 em relação $1,0 \mathrm{Mg} \mathrm{m}^{-3}$

\begin{tabular}{|c|c|c|c|c|c|}
\hline \multirow{2}{*}{ Corretivos } & \multirow{2}{*}{ Equação } & \multirow{2}{*}{$\mathrm{R}^{2}$} & \multicolumn{2}{|c|}{ Densidades $\left(\mathrm{Mg} \mathrm{m}^{-3}\right)$} & \multirow{2}{*}{$\begin{array}{c}\text { Redução } \\
(\%) \\
\end{array}$} \\
\hline & & & 1,00 & 1,60 & \\
\hline \multicolumn{6}{|c|}{ Acúmulo de cálcio $\left(\mathrm{g}\right.$ vaso $\left.^{-1}\right)$} \\
\hline \multicolumn{6}{|c|}{ Sem cultivo } \\
\hline Gesso & $Y=1,80-1,63 x+0,38 x^{2}$ & $0,99 *$ & 0,55 & 0,16 & 70 \\
\hline Calcário & $Y=1,97-1,83 x+0,438 x^{2}$ & $0,99 *$ & 0,58 & 0,16 & 72 \\
\hline Silicato & $Y=2,45-2,42 x+0,63 x^{2}$ & $0,93 *$ & 0,66 & 0,19 & 71 \\
\hline Calcário + gesso & $Y=2,41-2,08 x+0,438 x^{2}$ & $0,96^{*}$ & 0,77 & 0,20 & 74 \\
\hline Silicato + gesso & $Y=4,341-4,97 x+1,5 x^{2}$ & $0,97^{*}$ & 0,87 & 0,23 & 73 \\
\hline \multicolumn{6}{|c|}{ Com cultivo } \\
\hline Gesso & $Y=-1,73+3,27 x-1,25 x^{2}$ & $0,97^{*}$ & 0,29 & 0,30 & +3 \\
\hline Calcário & $Y=-2,56+4,54 x-1,69 x^{2}$ & $0,86^{*}$ & 0,29 & 0,38 & +31 \\
\hline Silicato & $Y=-0,60+1,43 x-0,44 x^{2}$ & $0,87^{*}$ & 0,39 & 0,56 & +43 \\
\hline Calcário + gesso & $Y=-3,67+6,37 x-2,38 x^{2}$ & $0,93 *$ & 0,33 & 0,43 & +30 \\
\hline Silicato + gesso & $Y=-4,264+7,55 x-2,88 x^{2}$ & $0,46^{*}$ & 0,41 & 0,44 & +7 \\
\hline \multicolumn{6}{|c|}{ Acúmulo de magnésio $\left(\mathrm{g}\right.$ vaso $\left.^{-1}\right)$} \\
\hline \multicolumn{6}{|c|}{ Sem cultivo } \\
\hline Gesso & $Y=0,106-0,05 x$ & $0,85^{*}$ & 0,06 & 0,03 & 50 \\
\hline Calcário & $Y=1,08-0,87 x+0,19 x^{2}$ & $0,99 *$ & 0,40 & 0,17 & 57 \\
\hline Silicato & $Y=0,70-0,42 x+0,06 x^{2}$ & $0,99 *$ & 0,34 & 0,19 & 44 \\
\hline Calcário + gesso & $Y=0,66-0,35 x+2 e-15 x^{2}$ & $0,99 *$ & 0,31 & 0,10 & 67 \\
\hline Silicato + gesso & $Y=0,93-0,72 x+0,13 x^{2}$ & $0,97 *$ & 0,34 & 0,11 & 67 \\
\hline \multicolumn{6}{|c|}{ Com cultivo } \\
\hline Gesso & $Y=-1,06+1,75 x-0,63 x^{2}$ & $0,94 *$ & 0,06 & 0,13 & +116 \\
\hline Calcário & $Y=0,78-0,73 x+0,25 x^{2}$ & $0,51^{*}$ & 0,30 & 0,25 & 16 \\
\hline Silicato & $Y=2,50-3,62 x+1,44 x^{2}$ & $0,99 *$ & 0,32 & 0,39 & 21 \\
\hline Calcário + gesso & $Y=0,20+0,11 x-0,06 x^{2}$ & $0,72 *$ & 0,25 & 0,22 & 12 \\
\hline Silicato + gesso & $Y=0,791-0,945 x+0,38 x^{2}$ & $0,31 *$ & 0,23 & 0,25 & 8 \\
\hline \multicolumn{6}{|c|}{ Acúmulo de enxofre $\left(\mathrm{g} \mathrm{vaso}^{-1}\right)$} \\
\hline \multicolumn{6}{|c|}{ Sem cultivo } \\
\hline Gesso & $Y=0,60-0,54 x+0,13 x^{2}$ & $0,99 *$ & 0,19 & 0,07 & 63 \\
\hline Calcário & $Y=0,88-1,02 x+0,32 x^{2}$ & $0,97 *$ & 0,18 & 0,07 & 61 \\
\hline Silicato & $Y=0,91-1,13 x+0,38 x^{2}$ & $0,92 *$ & 0,16 & 0,07 & 56 \\
\hline Calcário + gesso & $Y=1,03-1,10 x+0,31 x^{2}$ & $0,98 *$ & 0,24 & 0,06 & 75 \\
\hline Silicato + gesso & $Y=1,32-1,57 x+0,5 x^{2}$ & $0,99 *$ & 0,25 & 0,09 & 64 \\
\hline \multicolumn{6}{|c|}{ Com cultivo } \\
\hline Gesso & $Y=2,211-3,05 x+1,13 x^{2}$ & $0,81^{*}$ & 0,29 & 0,22 & 24 \\
\hline Calcário & $Y=1,48-1,80 x+0,63 x^{2}$ & $0,53^{*}$ & 0,31 & 0,21 & 32 \\
\hline Silicato & $Y=2,38-3,30 x+1,25 x^{2}$ & $0,70^{*}$ & 0,33 & 0,33 & 0 \\
\hline Calcário + gesso & $Y=2,07-2,61 x+0,94 x^{2}$ & $0,91^{*}$ & 0,40 & 0,30 & 25 \\
\hline Silicato + gesso & $Y=3,40-4,62 x+1,69 x^{2}$ & $0,79 *$ & 0,47 & 0,33 & 29 \\
\hline
\end{tabular}

*Significativo a $5 \%$ pelo teste $\mathrm{F}$ 


\section{CONCLUSÕES}

1. O cultivo prévio do capim-Marandu e o uso de corretivos mitigaram os efeitos negativos da compactação do solo sobre a nutrição da soja;

2.O uso de corretivos do solo contribuiu para o aumento no acúmulo de macronutrientes na parte aérea da soja, porém o incremento na compactação proporcionou uma diminuição no acúmulo de N, P, K, Ca, Mg e S;

3. A compactação do solo persistiu parcialmente mediante cultivo prévio do capim-marandu.

\section{AGRADECIMENTOS}

Ao Conselho de Desenvolvimento Científico e Tecnológico (CNPq), pela concessão da bolsa de estudos e à Fundação de Amparo a Pesquisa de Minas Gerais (FAPEMIG) pelo apoio financeiro.

\section{REFERÊNCIAS}

ALAKUKKU, L.; WEISSKOPF, P.; CHAMEN, W.C.T. Prevention strategies for field traffic-induced subsoil compaction: a review. Part I. Machine/soil interactions. Soil and Tillage Research, v.73, n. 01/02, p. 145-160, 2003.

AZEVEDO, D.M.P. et al. Atributos físicos e químicos de um Latossolo Amarelo e distribuição do sistema radicular da soja sob diferentes sistemas de preparo no cerrado maranhense. Revista Ciência Agronômica, v. 38, n. 01, p. 38-40, 2007.

CARVALHO-PUPPATTO, J. G.; BÜLL, L .T.; CRUSCIOL, C. A. C. Atributos químicos do solo, crescimento radicular e produtividade do arroz com a aplicação de escórias. Pesquisa Agropecuária Brasileira, v. 39, n. 12, p. 1213-1218, 2004.

CARVALHO, R. et al.. A. Dessorção de fósforo por silício em solos ácidos. Revista Brasileira Ciência do Solo, v. 24, p. 69-74, 2000.

CAIRES, E. F. et al. Soil acidity and aluminum toxicity as affected by surface liming and cover oat residues under a no-till system. Soil Use Management. v.24, n. 03, p. 302-309, 2008.

CASTRO FILHO, C.; MUZILLI, O.; PODANOSCHI, A. L. Estabilidade dos agregados do solo em função de sistema de plantio, rotações de culturas, preparo da amostra e teor de matéria orgânica. Revista Brasileira Ciência do Solo, v. 22, p. 527-538, 1998.

CORRÊA, J. C. et al. Alteração de atributos físicos em Latossolo com aplicação superficial de escória de aciaria, lama cal, lodos de esgoto. Revista Brasileira Ciência do Solo, v.33, n. 02 , p. 263-272, 2009.

CORRÊA, J. C.; MAUAD, M.; ROSOLEM, C. A. Fósforo no solo e desenvolvimento da soja influenciada pela adubação fosfatada e cobertura vegetal. Pesquisa Agropecuária Brasileira v. 39, n. 12, p. 1231-1237, 2004.

CORRÊA, J. B. D. et al. Concentração de macronutrientes na parte aérea do cafeeiro em diferentes graus de compactação e classes de solos. In: SIMPÓSIO DE PESQUISA DOS CAFÉS DO BRASIL, 2001, Vitória, ES. Anais... Vitória, 2001.1 CD-ROM.

EMPRESA BRASILEIRA DE PESQUISA AGROPECUÁRIA. Manual de análises químicas de solos, plantas e fertilizantes. Brasília: Embrapa Comunicação para transferência de tecnologia, 1999. $370 \mathrm{p}$.

FERREIRA, D. F. Sisvar 4.3. 2000. Disponível em: < http:// www.dex.ufla.br/danielff /sisvar>. Acesso em: 13 jul. 2007.

FOLONI, J. S.; CALONEGO, J. C.; LIMA, S. L. Efeito da compactação do solo no desenvolvimento aéreo e radicular de cultivares de milho. Pesquisa Agropecuária Brasileira v. 38, n. 08, p. 947-955, 2003.

GARCIA, R. et al. Forrageiras utilizadas no sistema integrado agricultura-pecuária. In: ZAMBOLIM, L.; SILVA, A. A. da; AGNES, E. L. (Ed.). Manejo integrado: integração agriculturapecuária. Viçosa, MG: UFV, 2004. p. 331-352.

GOEDERT, W. J.; SCHERMACK, M. J.; FREITAS, F. C. Estado de compactação do solo em áreas cultivadas no sistema de plantio direto. Pesquisa Agropecuária Brasileira, v. 37, n. 02, p. 223-227, 2002.

KLEIN, V. A.; LIBARDI, P. L. Densidade e distribuição do diâmetro dos poros de um Latossolo Vermelho, sob diferentes sistemas de uso e manejo. Revista Brasileira Ciência do Solo, v. 26, p. $857-867,2002$.

MALAVOLTA, E. Elementos de nutrição de plantas. Piracicaba: Agronômica Ceres. 1980. 251 p.

MALAVOLTA, E. Avaliação do estado nutricional da plantas: princípios e aplicações. 2. ed. Piracicaba: Potafos, 1997. 319 p.

MATIAS, S. S. R. et al. Atributos físicos de um Latossolo Vermelho submetido a diferentes usos. Revista Ciência Agronômica, v. 40, n. 03 p. 331-338, 2009.

NOVAIS, R. F.; SMITH, T. J. Fósforo em solo e em condições tropicais. Viçosa: UFV, 1999. 399 p.

OLIVEIRA, J. B. Pedologia Aplicada. 3. ed. Piracicaba: FEALQ, 2008. $592 \mathrm{p}$.

PEREIRA, H. S. et al. Silicon sources for Rice crops. Scientia Agraria, v. 61, n. 05, p. 522-528, 2004.

POZZA, A. A. et al. Retenção e dessorção competitivas de ânions inorgânicos em gibsita natural de solo. Pesquisa Agropecuária Brasileira, v. 42, n. 11, p. 1627-1633, 2007.

RIBEIRO, A. C.; GUIMARÃES, P. T. G.; ALVAREZ V., H. V. (Ed.) Recomendações para uso de corretivos e fertilizantes em Minas Gerais. $5^{a}$ Aproximação. Viçosa: Comissão de Fertilidade do Solo do Estado de Minas Gerais - CFSEMG, 1999. 359 p.

SANTOS, G. A.; CAMARGO, F. A. O. Fundamentos da matéria orgânica no solo: Ecossistemas tropicais e subtropicais. Porto Alegre: Gênesis, 1999. 518 p. 
SHIERLAW, J.; ALSTON, A. M. Effect of soil compaction on root growth an uptake of phosphorus. Plant and Soil, v. 77, n. 01, p. 15-26, 1984.

SILVA,A.J.N.;CABEDA,M.S.V.Compactaçãoecompressibilidade do solo sob sistemas de manejo e níveis de umidade. Revista Brasileira Ciência do Solo, v. 30, p. 921-930, 2006.

SILVA, R. H.; ROSOLEM, C. A. Influência da cultura anterior e da compactação do solo na absorção de macronutrientes em soja. Pesquisa Agropecuária Brasileira, v. 36, n. 10, p. 1269-1275, 2001.

Alterações de características do solo na implantação de um sistema de integração agricultura-pecuária leiteira. 2005. 130 f. Dissertação (Mestrado em Produção Vegetal) - Universidade Federal do Paraná, Curitiba, 2005. SOUSA, D. M. G. et al. Acidez do solo e sua correção. In: NOVAIS, R.F. et al. Fertilidade do solo. Viçosa: Sociedade Brasileira de Ciência do Solo, 2007. p. 206-274.

SOUZA, M. A. S. et al. Previous cultivation of palisade grass and soil correctives: influence on growth and yield of soybean cultivated under various soil compaction levels. Ciência \& Agrotecnologia, v. 35, n. 06, p. 1132-1140, 2011.

SOUZA, Z. M.; ALVES, M. C. Movimento de água e resistência à penetração em um Latossolo Vermelho distrófico de cerrado, sob diferentes usos e manejos. Revista Brasileira de Engenharia Agrícola e Ambiental, v. 07, n. 01, p. 18-23, 2003.

ZAMBRANO, M. et al. Characterization of "dregs" and grits from cellulose paste industry: Study for its application to acid soils. Afinidad, v. 60, n. 503, p. 16-25, 2003. 